\title{
Factor VII as a marker of hepatocellular synthetic function in liver disease
}

\author{
G. GREEN, L. POLLER, JEAN M. THOMSON, AND I. W. DYMOCK \\ From the Departments of Medicine and Haematology, University Hospital of South Manchester
}

SYNOPSIS Factor VII levels have been measured in 100 patients with liver disease following parenteral vitamin $\mathrm{K}_{1}$ therapy. There was good agreement between specific factor VII measurements and the one-stage prothrombin time apart from six patients with compensated cirrhosis in whom the prothrombin time was prolonged despite the presence of normal factor VII levels. A mean activity of $58 \%$ was found in patients with cirrhosis. Cirrhotic patients with features of hepatic decompensation had a significantly lower mean level of activity $(40 \%)$ than the 'contrast' patients with surgical obstruction of the major bile ducts $(93 \%)$. Patients with chronic active liver disease had moderate depression of factor VII levels and those with non-cirrhotic alcoholic liver damage had mean activities similar to the contrast group.

Factor VII levels could not be correlated with BSP retention but there was a correlation with serum albumin concentration.

It is concluded that the prothrombin time using the Quick test with a standardized thromboplastin showing good sensitivity to factor VII, eg, the Manchester reagent (BCT), provides a reliable index of coagulability in chronic liver disease, and specific factor VII assays are not indicated.

The liver is the major site of synthesis of many clotting factors (Roberts and Cederbaum, 1972) and it is not unexpected that reduced levels of these have been reported in liver disease (Owren, 1949; Hallen and Nilsson, 1964; Donaldson et al, 1969).

Biochemical tests of liver function have traditionally been used to assess and monitor the progress of patients with hepatic disorders. More recently, both broad-spectrum tests of blood clotting, ie, onestage prothrombin time and partial thromboplastin time and specific clotting factor measurements, particularly factor VII assays (Dymock et al, 1975), have proved of additional value in the diagnosis and assessment of liver disease. When measuring factor VII levels most workers have had to rely on combined assays such as the $\mathbf{P}$ and $\mathbf{P}$ test and Normotest (Hillenbrand and Sherlock, 1973; Henning and Yano, 1973) which are affected by deficiencies of other clotting factors, eg, factors II and $\mathrm{X}$ as well as factor VII. However, the availability of a naturally occurring factor VII deficient plasma in beagle dogs (Poller et al, 1971) has provided a standardized specific clotting factor VIl assay. Its importance in accurately reflecting the state of hepatic function in

Received for publication 8 April 1976 clinical situations, particularly acute hepatic failure, has recently been reported (Poller et al, 1971; Dymock et al, 1975).

This study assesses the levels of factor VII after a course of parenteral vitamin $K_{1}$ in patients with chronic hepatic disease. After the administration of this vitamin it is assumed that the level of factor VII and other prothrombin complex factors will relate solely to the liver's synthetic ability. Their correlation with other clinical and laboratory parameters of hepatic function is determined. An assessment is made of the genuine need for specific factor VII measurement in addition to the one-stage prothrombin time in chronic liver disease.

\section{Patients and methods}

One hundred consecutive patients admitted to the Liver Unit of this hospital had, in addition to routine haematological and biochemical tests, an evaluation of their levels of factor VII within 72 hours of completing an intravenous course of vitamin $\mathbf{K}_{1}$ therapy in a dose of $20 \mathrm{mg}$ daily. Factor VII assays were performed using a single batch of lyophilized plasma from congenital factor VII deficient beagle dogs (Poller et al, 1971). 
For an analysis of the results, patients were grouped diagnostically and the relationships between factor VII levels, serum albumin, one-stage prothrombin time, and bromosulphthalein retention were correlated.

Twenty-three patients who were subsequently shown to have surgical obstruction to the major bile ducts as a cause of their abnormal liver function were chosen as a 'contrast' group as hepatocellular function might be expected to be only minimally disturbed in these people, and in all cases there was a return to normal biochemical function after surgical relief of the obstruction.

\section{Results}

The final diagnoses reached in the 100 patients studied are shown in the table. All patients, in-

\begin{tabular}{lllll}
\hline Diagnosis & $\begin{array}{l}\text { No. of } \\
\text { patients }\end{array}$ & $\begin{array}{l}\text { Mean } \\
\text { FVII \% }\end{array}$ & $\begin{array}{l}\text { Mean } \\
\text { serum } \\
\text { albumin } \\
(g / l)\end{array}$ & $\begin{array}{l}\text { Mean } \\
P T(s)\end{array}$ \\
\hline $\begin{array}{l}\text { Obstructive jaundice } \\
\text { Cirrhosis }\end{array}$ & 23 & 93 & 36 & $13 \cdot 5$ \\
$\begin{array}{l}\text { Chronic active liver } \\
\text { disease }\end{array}$ & 41 & 58 & $31 \cdot 3$ & $18 \cdot 5$ \\
$\begin{array}{l}\text { Alcoholic liver } \\
\text { damage }\end{array}$ & 18 & 75 & 35 & $13 \cdot 0$ \\
\begin{tabular}{l} 
Total \\
\hline
\end{tabular} & 18 & 93 & 38 & $12 \cdot 5$ \\
\hline
\end{tabular}

Table Diagnostic groups studied illustrating numbers of patients, mean factor VII level, serum albumin concentration, and prothrombin time

cluding the 18 with non-cirrhotic alcoholic liver damage, had histological or surgical confirmation of the diagnosis.

\section{OBSTRUCTIVE JAUNDICE}

In seven of the 23 patients in this group the prothrombin time was prolonged, but in only four of these was the factor VII activity low (fig 1). The mean prothrombin time was 13.0 seconds and the mean factor VII activity $93 \%$. There was a normal mean serum albumin of $36 \mathrm{~g} / 1(3.6 \mathrm{~g} / 100 \mathrm{ml})$.

\section{CIRRHOSIS}

In 23 of the 41 patients with hepatic cirrhosis there was an alcoholic aetiology, There was no significant difference between the mean factor VII levels, prothrombin times, and serum albumin concentrations of the alcoholic and non-alcoholic cirrhotic patients. The mean factor VII level of the 41 patients was $58 \%$, which was significantly less than in the patients with biliary obstruction $(P<0.0001)$. The mean serum albumin of $31.3 \mathrm{~g} / 1$, although lower than that in obstructive jaundice, was still within the lower

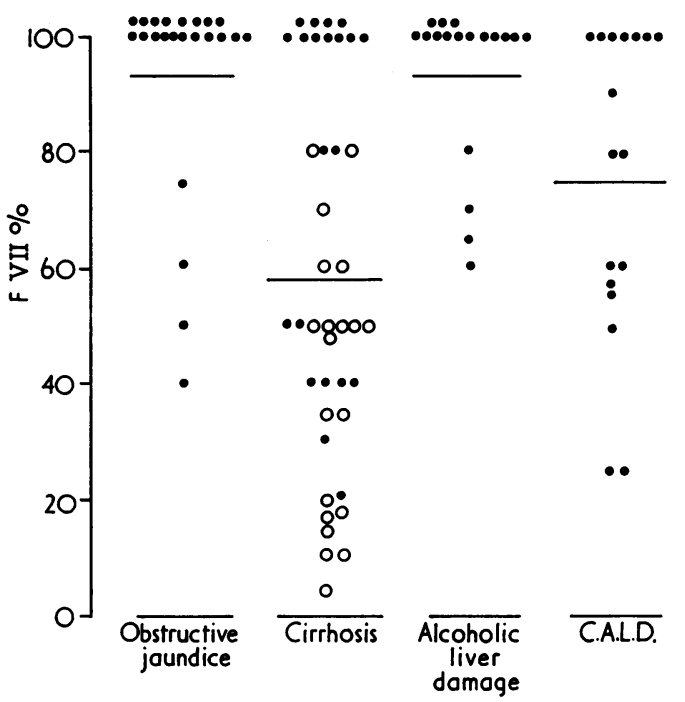

Fig 1 Factor VII levels in the patients studied: $0=$ 'hepatic decompensation' or coma; $C A L D=$ chronic active liver disease.

limit of our normal range. The mean prothrombin time of 18.5 seconds was significantly prolonged $(P<0.001)$. Twenty-three patients had a proge thrombin time greater than 13.5 seconds, but five of these had a normal level of factor VII.

Nineteen of the 41 patients with histologically proven cirrhosis exhibited features of hepatocellular decompensation, ie, hepatic coma or precoma and/or ascites, oedema, and hyponatraemia. This group was of particular interest. The mean factor VII level of these patients, $40 \%$, was significantly less than that of both the obstructive patients and the 'compensated' cirrhotics $(P<0.0001$ and $<0.0005$ respectively). The mean prothrombin time (19.5 seconds) was also significantly prolonged. A similar difference was likewise observed in the serum albumin concentrations (fig 2). The correlation of prothrombin time and factor VII was absolute, both tests being abnormal in all cases.

Of the compensated cirrhosis patients, 16 showed an abnormal prothrombin time but only 10 had a low factor VII activity. The shorter mean prothrombin time (15.5 seconds) in the compensated of group was associated with a higher mean factor $\tilde{O}$ VII activity $(74 \%)$ than in the decompensated $\underset{\omega}{N}$ cirrhotic patients. In compensated cirrhosis the agreement of these two tests was not as close as in the decompensated group. Sixteen of 22 patients demonstrated agreement between the two tests but six $\stackrel{\mathcal{C}}{?}$ had normal factor VII activity although the pro- $\square$ thrombin time was prolonged. Follow-up tests in four of these six showed a shortening of the pro- 


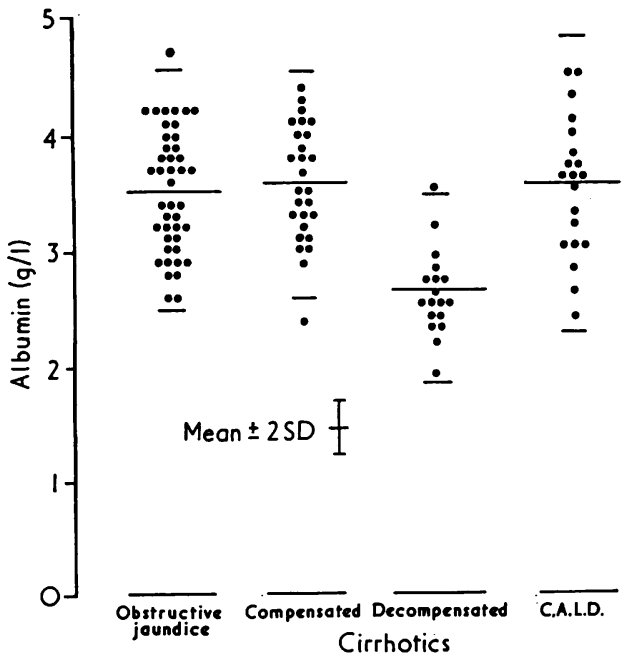

Fig 2 Serum albumin concentrations in patients with obstructive jaundice, cirrhosis, and chronic active liver disease (CALD).

thrombin time, which in three patients was to the normal range. The implications in terms of assessment of hepatocellular synthesis are discussed later. The mean prothrombin time of the decompensated cirrhotics who survived was 20.0 seconds and of those who died 22.5 seconds. This difference was not significant. The corresponding mean factor VII levels were $49 \%$ and $30 \%$ respectively and were again not statistically different.

CHRONIC ACTIVE LIVER DISEASE (CALD)

Eleven of the 18 patients in this group had reduced levels of factor VII, the mean being $75 \%$, which was significantly less than that of the patients with biliary obstruction $(P<0.01)$.

The mean serum albumin of $35 \mathrm{~g} / \mathrm{l}$ was not significantly below that of the 'contrast' group (P > 0.5). Compared with the cirrhotics there was a slight difference in the mean serum albumin levels of the two groups although in CALD the level was within the normal range. The mean factor VII levels were significantly lower in the cirrhotic patients $(P<$ 0.05 ); this was further reflected in the more prolonged one-stage prothrombin time of the cirrhotics. Eight patients had a prolongation of the prothrombin time associated with reduced factor VII levels.

NON-CIRRHOTIC ALCOHOLIC LIVER DAMAGE The average factor VII level of these patients was $93 \%$. The mean prothrombin time of 13.5 seconds was not significantly different from the values observed in the 'contrast' group. Only three patients

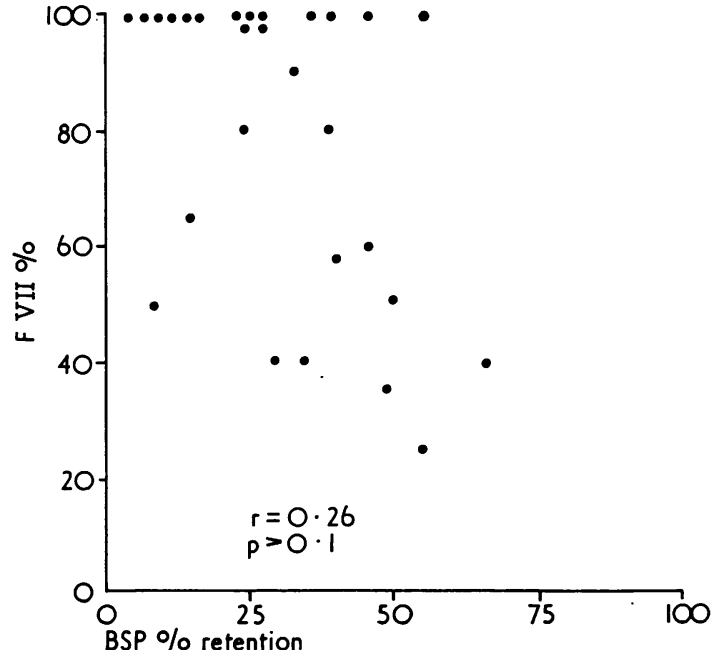

Fig 3 Correlation of factor VII levels and bromsulphophthalein $(B S P)$ retention at 45 minutes.

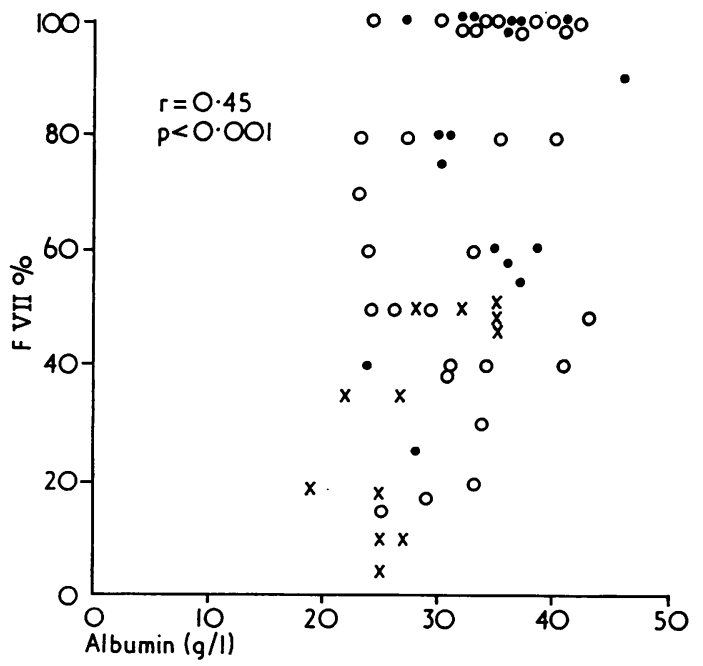

Fig 4 Correlation of factor VII levels and serum albumin concentrations. $C A L D ; \bigcirc$ compensated cirrhosis; $\times$ decompensated cirrhosis.

showed an appreciable prolongation of the prothrombin time and in each case this was associated with a low factor VII. The mean albumin concentration was $38 \mathrm{~g} / \mathrm{l}$.

RELATIONSHIP BETWEEN FACTOR VII LEVELS AND OTHER TESTS OF LIVER FUNCTION

Bromsulphophthalein retention (fig 3) Although there appeared to be some correlation between BSP retention and factor VII in the 34 patients in whom 


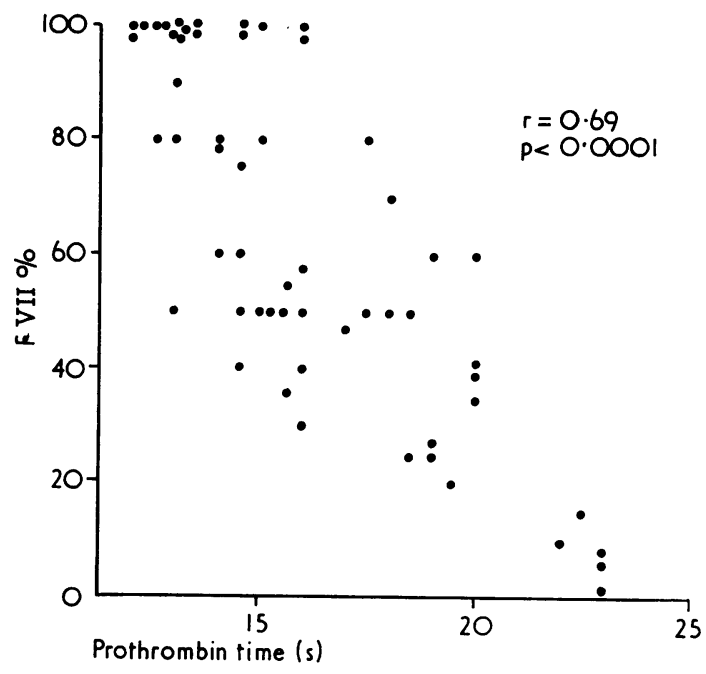

Fig 5 Correlation of factor VII levels and one-stage prothrombin time (seconds).

both parameters had been measured, this relationship did not reach statistical significance $(P>0 \cdot 1)$.

Serum albumin (fig 4) There was a correlation between factor VII levels and serum albumin concentrations $(\mathrm{P}<0.001)$. However, this correlation was not absolute, and 16 patients with low factor VII levels had serum albumin values above the lower limit of the normal range for this laboratory. Only two patients with a low serum albumin gave normal factor VII activity.

Prothrombin time (fig 5) The correlation coefficient between the prothrombin time and factor VII levels was significant $(\mathrm{P}<0.0001)$. However, this relationship, as detailed previously, was not a constant feature in the compensated cirrhotic patients. The results of the prothrombin time, factor VII levels, and serum albumin concentrations are summarized in the table.

\section{Discussion}

The emphasis in this study has been on factor VII levels as a marker of hepatocellular synthesis in patients with chronic liver disease, following the administration of parenteral vitamin $K_{1}$.

Factor VII, which is synthesized in the liver, has a short plasma half-life compared to other clotting factors, being in the region of two hours (Loeliger $e t$ $a l, 1964)$. It also provides a more specific measure of synthetic ability, being less affected by other disease mechanisms such as inflammation, disseminated intravascular coagulation, and accelerated fibrino- lysis, which may be present in liver disease. In practice, depressed factor VII activity tends to be the earliest detectable coagulation change accompanying severe acute hepatocellular disturbance and appears $\stackrel{\vec{\rho}}{\stackrel{F}{ }}$ to correlate most clearly with prognosis (Dymock et $a l, 1975)$, but this study shows the assay to be of less value in chronic liver disease.

In the group of patients with major bile duct $\frac{\mathbb{d}}{\alpha}$ obstruction, the great majority had normal factor i VII levels and prothrombin times following vitamin os $\mathrm{K}_{1}$ administration. These patients provided a useful contrast group. In the chronic active liver $\overrightarrow{\vec{\omega}}$ disease patients a substantial minority of subjects $\stackrel{\omega}{\mathscr{D}}$ had residual coagulation disturbance after vitamin $\frac{0}{8}$ $\mathrm{K}_{1}$ shown by both prolonged prothrombin time and i reduced factor VII levels. This indicated gross $\stackrel{\bullet}{\bullet}$ impairment of hepatic synthetic ability as suspected $\overrightarrow{\vec{\theta}}$ from routine biochemical tests such as serum albumin.

Patients with hepatic cirrhosis had markedly re- 을 duced levels of factor VII and prolonged prothrombin times. Levels in cirrhotics were significantly lower than in the chronic active group. This difference was due to a failure to respond to vitamin $K_{1}$ administration by approximately half of these patients, most of whom had decompensated cin rhosis.

Patients with liver cell damage secondary to ex. cessive alcohol consumption showed virtually n impairment of factor VII synthesis following parenteral vitamin $\mathrm{K}_{1}$. The 72-hour period of abstinence which accompanied the treatment may also have contributed to this observation.

Although the significance and possible importance of specific factor VII studies in liver disease have been appreciated in the assessment of liver synthetic reserve, there has been little practical opportunity to assess the value because of the lack of adequate factor VII deficient plasma substrate for the assays. Severe congenital deficiency of factor VII in man, 3 . suitable for supplying plasma substrate for the assay, $\delta$ is extremely rare. An artificial factor VII substrate is difficult to prepare. The discovery of a congenitally $\bigcirc$ severe defect of factor VII in a colony of beagle dogs has facilitated limited clinical studies which have o shown that the assay is of great value in assessing the prospects of survival in acute liver failure 0 (Dymock et al, 1975). As supplies of VII deficient N plasma are severely restricted it was important to $\underset{\mathrm{C}}{\mathrm{N}}$ evaluate if any important contribution was made by specific factor VIl assays in the management of patients with chronic liver disease. The conventional $\frac{0}{\varnothing}$ routine coagulation test for assessing liver function $\stackrel{0}{\rightarrow}$ is the prothrombin time. In Britain a standardized thromboplastin reagent, Manchester Comparative Reagent (BCT), possessing good sensitivity to 
factor VIl is usually employed in this test (Poller et al, 1971; Zucker et al, 1970). We have therefore compared the relative value of the specific factor VII assay with the broad-spectrum test, the prothrombin time using BCT, in the various groups with chronic liver disease. There was a suggestion that the factor VII assay gives an earlier index of responsiveness to $K_{1}$ therapy than the prothrombin time. Where a prolonged prothrombin time was associated with a low factor VIl activity after vitamin $K_{1}$ poor hepatic synthetic reserve might be inferred. It is reassuring that in only a few of the compensated cirrhotics were there discrepancies between the factor VII and prothrombin time results. In these cases the prothrombin time using BCT appears to be a better measure of the residual coagulation abnormality following the response to vitamin $K_{1}$ than specific factor VII assays.

In patients with obstructive jaundice, chronic active liver disease, and decompensated cirrhosis, the factor VII assay provides no additional information to the prothrombin time.

In those patients with decompensated cirrhosis, the failure of response to vitamin $K_{1}$ was demonstrated equally by both tests, and no additional information of the prothrombin time was provided by a specific factor VII assay to either classification of severity or prognosis. Both the prothrombin time test and the factor VII levels were, however, significantly more abnormal in the decompensated group than in the compensated cirrhotics.

There was no close correlation between factor VII levels and BSP retention. As the latter test is more closely linked to excretory function of the liver it suggests that impairment of the two functions need not follow a constant relationship. There was a good correlation of factor VII and albumin level but the wide scatter of the normal range of albumin concentrations plus the effect of other system disease, eg, renal, on its levels limits its sensitivity as a marker of liver synthesis.

It may be concluded that in chronic liver disease there are no advantages in performing the specific factor VII assay in addition to the prothrombin time using BCT. It must not be assumed, however, that this will be the case if the prothrombin time is performed with a different type of thromboplastin reagent, as it has been established that the BCT possesses greater sensitivity to factor VII than commercial thromboplastin reagents used in the Quick test (Zucker et al, 1970; Poller et al, 1971). It is thus reassuring that this study indicates that the conventional coagulation test available to all British hospitals provides adequate assessment of hepatic clotting factor synthesis in patients with chronic liver disease.

We thank Professor J. M. Evanson for provision of clinical facilities and S. R. Armitage for technical assistance.

\section{References}

Donaldson, G. W. K., Davies, S. H., Darg, A. and Richmond, J. (1969). Coagulation factors in chronic liver disease. J. clin. Path., 22, 199-204.

Dymock, I. W., Tucker, J. S., Woolf, I. L., Poller, L., and Thomson, J. M. (1975). Coagulation studies as a prognostic index in acute liver failure. Brit. J. Haemat., 29, 385-395.

Hallen, A. and Nilsson, I. M. (1964). Coagulation studies in liver disease. Thrombos. Diathes. haemorrh. (Stuttg.), 11, 51-63.

Henning, H. and Yano, M. (1973). Significance and practability of Normotest in diagnosis of chronic liver diseases. Scand. J. Gastroent., Supplement, 19, 119-123.

Hillenbrand, P. and Sherlock, S. (1973). Use of Normotest and Thrombotest, coagulation tests in hepatocellular disease. Scand. J. Gastroent., Supplement, 19, 125-131.

Loeliger, E. A., Van der Esch, B., and Mattern, M. J. (1964). The biological disappearance rate of prothrombin, factors VII, IX and $\mathbf{X}$ from plasma in hypothyroidism, hyperthyroidism, and during fever. Thrombos. Diathes. haemorrh. (Stuttg.), 10, 267-277.

Owren, P. A. (1949). The diagnostic and prognostic significance of plasma prothrombin and factor $\mathrm{V}$ levels in parenchymatous hepatitis and obstructive jaundice. Scand. $J$. clin. Lab. Invest., 1, 131-140.

Poller, L., Thomson, J. M., Sear, C. H. J., and Thomas, W. (1971). Identification of a congenital defect of factor VII in a colony of beagle dogs: the clinical use of the plasma. J. clin. Path., 24, 626-632.

Roberts, H. R. and Cederbaum, A. I. (1972). The liver and blood coagulation: physiology and pathology. Gastroenterology, 63, 297-320.

Zucker, S., Cathey, M. H., Sox, P. J., and Hall, E. C. (1970). Standardization of laboratory tests for controlling anticoagulant therapy. Amer. J. clin. Path., 53, 348-354. 